\title{
On the SU(2)-Higgs Phase Transition
}

\author{
Isabel Campos ${ }^{\mathrm{a} *}$ \\ a Departamento de Física Teórica, Universidad de Zaragoza, \\ Plaza San Francisco s/n, 50009 Zaragoza, Spain
}

We investigate the properties of the Confinement-Higgs phase transition in the SU(2)-Higgs model. The system is shown to exhibit a transient behavior up to $L=24$ along which, the order of the phase transition cannot be discerned. In order to get a global vision on the problem, we have introduced a second (next-to-nearest neighbors) gauge-Higgs coupling $\left(\kappa_{2}\right)$. On this extended parameter space we find a line of phase transitions becoming increasely weak as the standard case is approached $\left(\kappa_{2}=0\right)$. From the global behavior on this parameter space we conclude that the transition is also first order in the standard case.

\section{INTRODUCTION}

The Higgs sector of the SM can be approximated by the $\mathrm{SU}(2) \otimes \mathrm{U}(1)$ Higgs model. If as a first approximation one neglects the $\mathrm{U}(1)$ gauge coupling $\left(g_{\mathrm{U}(1)} \approx \sin \theta_{W} g_{\mathrm{SU}(2)}\right)$ one has to deal with the $\mathrm{SU}(2)$-Higgs model:

$$
\begin{aligned}
S_{\lambda}= & \beta \sum_{p}\left[1-\frac{1}{2} \operatorname{Tr} U_{p}\right]+\sum_{x} \Phi^{\dagger}(x) \Phi(x) \\
& +\lambda \sum_{x}\left[\Phi^{\dagger}(x) \Phi(x)-1\right]^{2} \\
& -\frac{1}{2} \kappa_{1} \sum_{x, \mu} \operatorname{Tr} \Phi^{\dagger}(x) U_{\mu}(x) \Phi(x+\mu)
\end{aligned}
$$

This model was extensively studied in the eighties 1.22. A phase transition (PT) line separates a region where the scalar particles are confined from another region where the spectrum consists of the W's bosons and the Higgs particle. The PT ends at some finite point of the parameter space, being both regions analytically connected. Concerning the order of the PT line, the model exhibits a second order PT with mean field exponents in the limit $\beta \rightarrow \infty$,pure $\lambda \Phi^{4}$ theory. At the endpoint the transition seems to be second order with mean field exponents, [3], however, this issue has still to be clarified. In the scaling region $(\beta \approx$ $2.5)$ and for small and intermediate values of $\lambda$ the PT is distinctly first order, getting increasely

\footnotetext{
*Supported by a Fellowship from the Ministerio de Educación y Cultura Español.
}

weak as $\lambda$ is increased. In particular, in the limit $\lambda=\infty$, which is equivalent to work with fixed modulus of the Higgs field, the PT is commonly believed to be first order though extremely weak. However the numerical simulations up to date are not conclusive.

\section{THE MODEL}

To improve on the statistics we have first studied the model with action $S_{\infty}$ up to $L=24$, being the results compatible, both, with a very weak first order PT and with a continuous one. In order to get a more conclusive answer without going to prohibitive large lattice sizes we have added a second positive gauge-Higgs coupling $\kappa_{2}$ connecting next-to-nearest neighbors on the lattice. The new action reads:

$$
\begin{aligned}
S= & S_{\infty}-\frac{1}{4} \kappa_{2} \sum_{x, \mu<\nu} \operatorname{Tr} \Phi^{\dagger}(x)\left[U_{\mu}(x) U_{\nu}(x+\mu)\right. \\
& \left.+U_{\nu}(x) U_{\mu}(x+\nu)\right] \Phi(x+\mu+\nu)
\end{aligned}
$$

Heuristically speaking, the effect of the new coupling is to reinforce the PT, but it should not change its order since the symmetry properties remain unchanged. This coupling will also be used as a parameter to monitorize the weakening mechanism of the PT.

\section{RESULTS}

On the parameter space $\left(\beta, \kappa_{1}, \kappa_{2}\right)$ we consider the plane $\beta=2.3$. On this plane there is a PT line 
Table 1

Latent heat and change in the action. The values at $\kappa_{1}=0.3$ are only upper bounds.

\begin{tabular}{lrrr}
\hline & $\Delta E_{1}$ & $\Delta E_{2}$ & $\Delta S$ \\
\hline$\kappa_{1}=0$ & - & $0.0366(8)$ & $0.0134(12)$ \\
$\kappa_{1}=0.02$ & $0.0162(6)$ & $0.0347(5)$ & $0.0137(13)$ \\
$\kappa_{1}=0.1$ & $0.0162(7)$ & $0.0345(9)$ & $0.0094(10)$ \\
$\kappa_{1}=0.2$ & $0.0179(7)$ & $0.0201(8)$ & $0.0078(12)$ \\
$\kappa_{1}=0.3$ & $\approx 0.006$ & $\approx 0.012$ & $\approx 0.0026$ \\
\hline
\end{tabular}

$\kappa_{2}^{c}\left(\kappa_{1}\right)$ along which, we shall study the existence of latent heat and the behavior of the specific heat with the volume. We worked at fixed values $\kappa_{1}=$ $0,0.02,0.1,0.2,0.3$ and $\kappa_{2}=0$ (standard case). To locate the critical line we sought for the maximum of the specific heat $\left(C_{v}^{\max }(L)\right)$. We have used the Spectral Density Method [1 to locate the couplings where to measure. The phase diagram we found with this criterium is plotted in Fig. 11 for $L=12$.

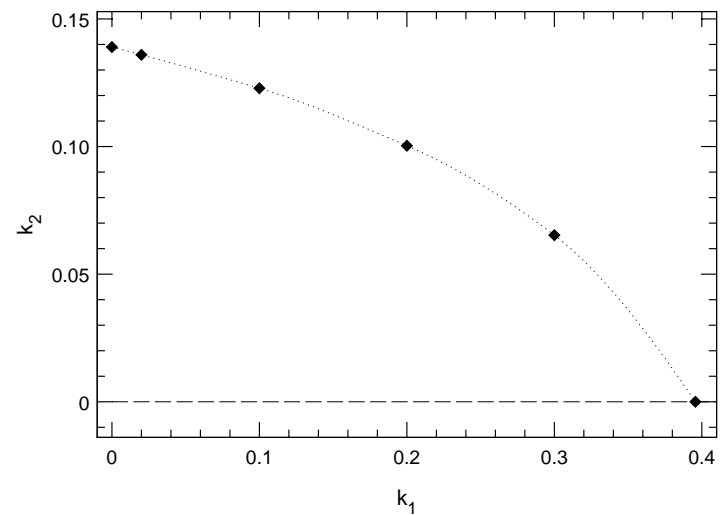

Figure 1. Phase Diagram obtained from the MC simulation with $L=12$.

\subsection{Latent Heat}

The PT line $\kappa_{2}^{c}\left(\kappa_{1}\right)$ turns out to be first order. In Fig. 2 the energy distribution for $L=12$ is plotted for $\kappa_{1}=0.02,0.1,0.2$ and 0.3 . We observe that the latent heat $\Delta E(L)$ is no longer measurable at $\kappa_{1}=0.3$, and one has to go to $L=20$ lattice to see the first evidences about the existence of latent heat at this value of the couplings. This evidences the weakening of the PT as the limit $\kappa_{2}$ $=0$ is approached. Weak first order PT appear often in literature [5] and they can be defined like first order PT with small discontinuities. They are characterized by a transient behavior during which the lattice size $L$ is much smaller than the correlation length at critical point, $\xi_{c}$. Along this regime the $\mathrm{PT}$ transition behaves like a second order one since $\xi_{c}$ is effectively infinite. Once the asymptotic regime is reached, $L \geq \xi_{c}$, the first order character is evidenced appearing the typical two-peak structure in the energy distribution. In Table 1 we report the results obtained for $\Delta E(\infty)$ by fitting the values of $\Delta E(L)$ to $1 / \mathrm{L}^{4}$.

At $\kappa_{2}=0$ (standard case) we have no distinct observations of two-peak energy distributions up to $L=24$ (Fig. 3).

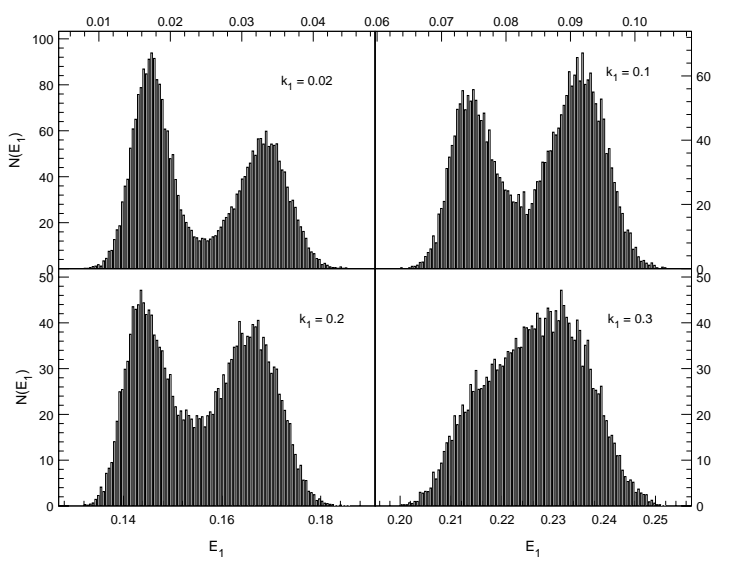

Figure 2. $\mathrm{E}_{1}$ distributions for several values of $\kappa_{1}$ in $L=12$. 


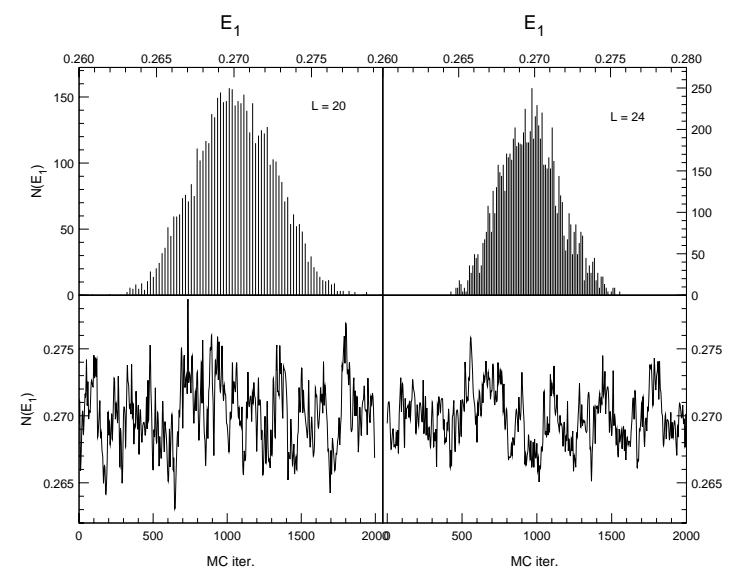

Figure 3. $\mathrm{E}_{1}$ distributions and MC evolution at $\kappa_{2}=0$ in $L=20$ and $L=24$

\subsection{Specific heat}

We now address the question of the behavior of the specific heat, $C_{v}(L)$ along the transient region of a weak first order phase transition. We use, as a technical tool, a pseudo $\nu$ exponent in order to discern whether or not the asymptotic regime has been reached since we expect to measure $\nu$ compatible with 0.25 (i.e. $1 /$ d) in the asymptotic region. We have measured the evolution of $C_{v}(L)^{\max }$ with the volume along the PT line. This maximum is expected to scale with a pseudo $\alpha / \nu$ index, and we obtain $\nu$ from the relation $\alpha=2-\nu d$.

In Fig. 1 we plot $\mathrm{C}_{v}^{\max }(L)$ relative to $\mathrm{C}_{v}^{\max }(6)$ as a function of the lattice size. The slope of the segment joining the values of $\mathrm{C}_{v}^{\max }(L)$ in consecutive lattices gives the pseudo $\alpha / \nu$ exponent. The values for $\nu$ are compatible with 0.25 at $\kappa_{1}=0.02$, 0.1 and 0.2 for all the volumes we compare. However the transition at $\kappa_{1}=0.3$ seems to be much weak. We do not have evidences of asymptoticity in $\mathrm{C}_{v}^{\max }(L)$ till $\mathrm{L}=20$, as could be expected from the energy distributions. At $\kappa_{2}=0, \mathrm{C}_{v}^{\max }(L)$ behaves qualitatively in the same way.

\section{CONCLUSIONS}

We have studied an extended parameter space in order to get insight on the nature of the $\mathrm{SU}(2)$ Higgs PT with $\lambda=\infty$ at $\mathrm{T}=0$. We have found

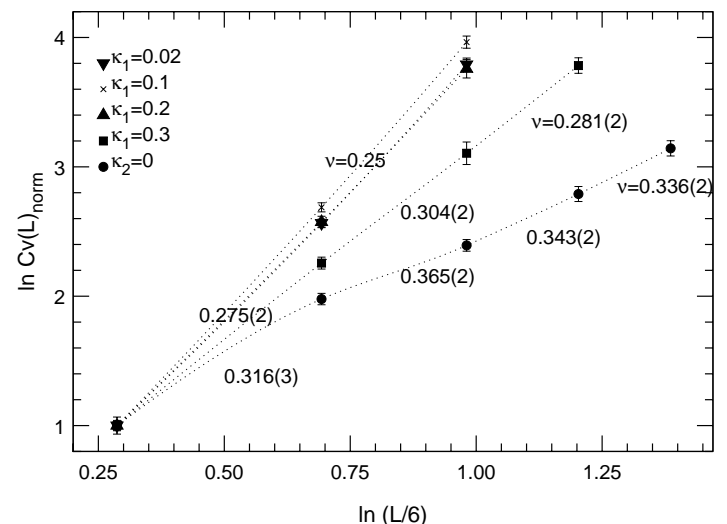

Figure 4. $\mathrm{C}_{v}^{\max }$ for the various $\kappa_{1}$ values and $\kappa_{2}=$ 0 . We have normalized the values with respect to $\mathrm{C}_{v}^{\max }(8) / \mathrm{C}_{v}^{\max }(6)$.

a line of first order phase transitions getting increasely weak as the standard case $\left(\kappa_{2}=0\right)$ is approached. The results in this limit are compatible with a continuous PT too. However, from the behavior in the global parameter space of the specific heat we conclude that we are in the transient region of a first order phase transition up to $L=24$.

I thank L.A. Fernández and A. Tarancón for comments and advice.

\section{REFERENCES}

1. C.B. Lang, C. Rebbi and M.A. Virasoro. Phys. Lett. B104, p 294(1981)

2. W. Langguth, I. Montvay and P. Weisz. Nuc. Phys. B277, p 11(1986)

3. D.J.E. Callaway and R. Petronzio Nuc. Phys. B267, p 253 (1986)

4. A. M. Ferrenberg and R. Swendsen. Phys. Rev. Lett. 61, p 2635(1988)

5. L.A. Fernández, M.P. Lombardo and J.J. Ruiz-Lorenzo. Phys. Lett. B277, p 485(1992) 